NASA

Technical Memorandum 107642

$$
\text { p.ll }
$$

USAAVSCOM

Technical Report 92-B-010

\title{
A DEMONSTRATION OF PASSIVE BLADE TWIST CONTROL USING EXTENSION-TWIST COUPLING
}

\author{
Renee C. Lake \\ Mark W. Nixon \\ Matthew L. Wilbur \\ Jeffrey D. Singleton \\ Paul H. Mirick
}

June 1992

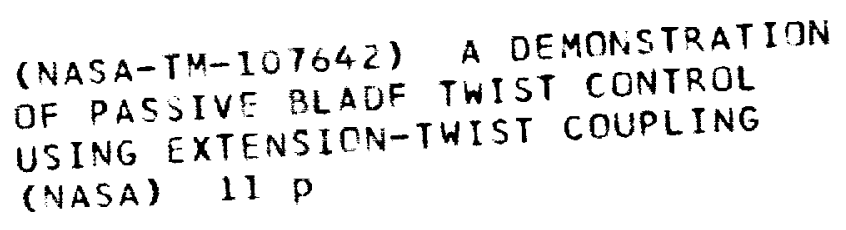

N92-31155

Unclas

$63 / 39 \quad 0115122$
National Aeionautics and

Space Administration

Langley Research Center

Hampton, Virginia 23665

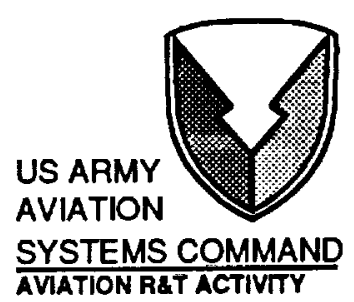




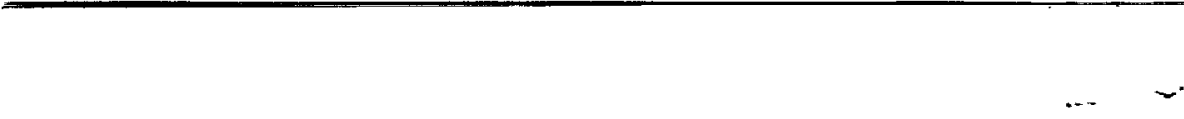




\title{
A DEMONSTRATION OF PASSIVE BLADE TWIST CONTROL USING EXTENSION-TWIST COUPLING
}

\author{
R. C. Lake, M. W. Nixon, M, L. Wilbur, J. D. Singleton, \\ and P. H. Mirick \\ U. S. Army Aerostructures Directorate \\ NASA Langley Research Center \\ Hampton, Virginia
}

\begin{abstract}
The results from a study aimed at improving the aerodynamic characteristics of composite tiltrotor blades through the use of extension-twist elastic coupling are presented. A set of low-twist model-scale helicopter rotor blades was manufactured from existing molds, with a view towards demonstrating the passive blade twist control concept. Hover testing of the set of rotor blades was conducted with the primary goal of measuring the change in blade twist as a function of rotor speed. The blades were spun through the $0-800 \mathrm{rpm}$ range, with a corresponding sweep of collective pitch at $100 \mathrm{rpm}$ intervals to determine the effect on the blade elastic twist obtained. Data were obtained in hover for both an unballasted and ballasted blade configuration in atmospheric conditions, where maximum twist changes of $2.54^{\circ}$ and $5.24^{\circ}$ were respectively observed. These results compared well with those obtained from a detailed finite-element analysis model of the rotor blade, which yielded maximum twists of $3.01^{\circ}$ and $5.61^{\circ}$ for the unballasted and ballasted blade configurations, respectively. A ballasted blade configuration was similarly tested in a near-vacuum condition as a means of determining the effect of aerodynamic contributions to the total twist obtained. Aerodynamic-induced effects on blade elastic twist were found to be minimal, with a maximum twist difference of $0.17^{\circ}$ observed between the two test environments. The effect of sweeping collective pitch on the blade elastic twist was also demonstrated to be minimal, as no appreciable degradation in twist was observed at increased collective pitch angles.
\end{abstract}

\section{Symbols}

$b \quad$ number of blades

$C_{T} \quad$ rotor thrust coefficient, $T / \rho \pi(R)^{2}(\Omega R)^{2}$

$C_{11} \quad$ extensional stiffness, $l b$

$C_{22}$ transverse shear (chordwise) stiffness, $l b$

$C_{33}$ transverse shear (flapwise) stiffness, $l b$

$C_{44}$ torsional stiffness, $l b$-in ${ }^{2}$

$C_{55}$ flapwise bending stiffness, $l b-i^{2}$

$C_{66} \quad$ chordwise bending stiffness, $l b-i n^{2}$

$C_{14}$ extension-torsion coupling stiffness, $l b$-in

\begin{tabular}{|c|c|}
\hline 5 & $\begin{array}{l}\text { chordwise shear/flapwise bending coupling } \\
\text { stiffness, } l b \text {-in }\end{array}$ \\
\hline 36 & $\begin{array}{l}\text { flapwise shear/chordwise bending coupling } \\
\text { stiffness, } l b \text {-in }\end{array}$ \\
\hline 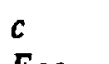 & $\begin{array}{l}\text { stiffness, } b \text { - } i n \\
\text { nominal blade chord, in }\end{array}$ \\
\hline 11 & extension modulus (spanwise), lb/in ${ }^{2}$ \\
\hline & extension modulus (chordwise), $l b / \mathrm{in}^{2}$ \\
\hline 12 & shear modulus, $l b / i n^{2}$ \\
\hline & $\begin{array}{l}\text { torsional mass moment of inertia per unit } \\
\text { length about blade feathering axis, } l b-s^{2}\end{array}$ \\
\hline$R$ & $\begin{array}{l}\text { rotor radius, in (converted to } f t \text { for calculation } \\
\text { of } C_{T} \text { ) }\end{array}$ \\
\hline & $\begin{array}{l}\text { spanwise distance along blade radius from } \\
\text { center of rotation, in }\end{array}$ \\
\hline & material shear strength, $l b / \mathrm{in}^{2}$ \\
\hline & rotor thrust, $l b$ \\
\hline & $\begin{array}{l}\text { material axial strength in compression, } \\
\mathrm{lb} / \mathrm{in}^{2}\end{array}$ \\
\hline & material axial strength in tension, $l b / i^{2}$ \\
\hline & $\begin{array}{l}\text { material transverse strength in compression, } \\
l b / \text { in }^{2}\end{array}$ \\
\hline & material transverse strength in tension, $l b /$ in $^{2}$ \\
\hline & $\begin{array}{l}\text { change in blade elastic twist at tip, positive } \\
\text { nose up, degrees }\end{array}$ \\
\hline & $\begin{array}{l}\text { blade collective pitch angle at } r / R=0.0 \text {, } \\
\text { degrees }\end{array}$ \\
\hline$\theta_{1}$ & $\begin{array}{l}\text { twist angle built into blade, positive nose } \\
\text { degrees }\end{array}$ \\
\hline & Poisson's ratio \\
\hline 0 & $\begin{array}{l}\text { density, } l b / \text { in }^{3} \text { (converted to slugs } / f^{3} \text { for } \\
\text { calculation of } C_{T} \text { ) }\end{array}$ \\
\hline & $\begin{array}{l}\text { solidity, } b c / \pi R \\
\text { rotor rotational velocity, } \mathrm{rad} / \mathrm{sec}\end{array}$ \\
\hline
\end{tabular}

\section{Introduction}

Tiltrotor aircraft are designed to operate in both helicopter and airplane modes of flight. This operational flexibility results in several conflicting design requirements. One such design requirement, which has significant effects on aerodynamic performance, is the built-in twist of the rotor blade. Typically, the built-in twist is not optimum for either flight mode. 
Performance could be improved if it were possible to vary blade twist between the airplane and helicopter modes. Tiltrotor aircraft typically vary rotor speed by about 20 percent between flight modes $(100 \% \mathrm{rpm}$ in helicopter mode, $80 \% \mathrm{rpm}$ in airplane mode) which induces a rather substantial change in the centrifugal force. Thus, a design which allows blade twist to change passively as a function of this difference in centrifugal force would lead to increased performance for tiltrotor aircraft. This could be effectively accomplished with the implementation of extension-twist coupling in the tiltrotor blade spar design.

Given the complex, directional nature of composite materials, as well as the complicated structural design requirements associated with advanced tiltrotor blade designs, many problems arise in the development of extension-twist-coupled tiltrotor blades. Foremost among these is the lack of an extensive analytical/experimental database for elastically-coupled structures. As such, significant amounts of basic research directed towards developing a better understanding of the fundamental problems inherent in new design concepts are required. For example, the role of physical geometries in the behavior of elastically-coupled composite rotor blades has been addressed. In reference 1 , the effects of pre-twist on the extension, bending, and torsional behavior of anisotropic beams with nonhomogeneous, irregular cross-sections were investigated. The study focused on the variation of extension-twist coupling as a function of pre-twist magnitude, as well as the location of the pre-twist axis. Numerical results indicated that the torsional stiffness in the one and two-cell designs decreased for moderate levels of pre-twist, then increased for high pre-twist levels, when the pre-twist axis was held at the section centroid. Varying the location of the pre-twist axis affected the magnitude and directional nature of the extension-twist coupling such that the beam would untwist or further twist in response to an applied extension. The maximum negative extension-twist coupling (largest untwisting of the beam) occurred when the pre-twist axis was located near the centroidal axis.

The global response characteristics of general thinwalled anisotropic beams which exhibit shear and warping deformations were studied in reference 2. Results from an anisotropic beam finite-element analysis were experimentally verified with a set of static axial tests performed on different configurations of extensiontwist-coupled tubular beam structures to assess the influence of warping-prone cross-sectional geometries. Test results demonstrated that bending-related warping effects have significant influence on beam-like structures, and are amplified by the extension-twist coupling, while the effects of torsion-related warping were not found to be significant for practical rotor structures.

The static behavior of elastically-coupled composite beams undergoing large deflections was analytically and experimentally investigated in reference 3 . A set of flat, graphite/epoxy beam specimens, representative of long flexible blades, were fabricated and subjected to static tip loading to determine the effect of various types of elastic coupling. The types of coupling studied included bending-twist coupling and extension-twist coupling. A set of reference (uncoupled) beams were also tested. Results from test and analysis demonstrated that coupling effects can have a significant effect on the large deflection behavior of elastically-coupled beams. Specifically, the twist behavior of the bending-twist-coupled beams was shown to be sensitive to the magnitude and orientation of a rigid body-type rotation about the beam root. The twist behavior of extension-twist-coupled beams was observed to be fairly similar to the uncoupled beams as a result of the low axial force produced by the tip loading. Thus, an optimum demonstration of the coupling effects produced by extension-twist-coupled beams could not be obtained. However, these results represent an important contribution to the experimental database.

Additional problems associated with the design of practical extension-twist-coupled rotor blades include the determination of optimum twist distributions that may be realistically obtained from current composite material systems, such as that studied in reference 4 . Two extension-twist-coupled designs representative of a tiltrotor blade were developed based on theoretically optimum aerodynamic twist distributions, and indicated a general twist rate requirement in the $0.216-0.333$ degree/inch range. Test data obtained from static tensile and torsion tests conducted on a set of extension-twistcoupled circular tube designs verified that the twist deformation required for tiltrotor blade designs could be achieved using a $\left(\left[+20^{\circ} /-70^{\circ}\right]_{2}\right)_{s}$ laminate design. This design produced twist rates within a $0.384-0.487$ degree/inch range. This study was further expanded in reference 5 , where the optimum linear blade twist distributions of a typical tiltrotor blade were shown to be $-20^{\circ}$ in hover and $-42^{\circ}$ in forward flight, based on minimum horsepower requirements. These twist distributions represented a net savings in power of $6.1 \%$ for the hover mode and $6.5 \%$ for the forward flight mode. Next, a set of extension-twist-coupled tiltrotor blade designs with varying amounts of tip weight were developed to achieve maximum twist deformations within material design limits. The optimized designs demonstrated significant improvements in hover and forward flight performance. Maximum twist deformations of $19^{\circ}$ and $27.3^{\circ}$ were obtained for the $0 \%$ $100 \% \mathrm{rpm}$ range for two practical designs, resulting in twist deformations between $80 \%-100 \%$ rpm of $6.9^{\circ}$ and $9.8^{\circ}$, respectively. While these results indicate that passive blade twist control is viable and can enhance current tiltrotor blade performance, an experimental verification of the concept is a necessary prerequisite in the practical development of an extension-twist-coupled tiltrotor blade design.

The purpose of this paper is to present results from an analytical/experimental study which investigated the potential for passively changing blade twist through the use of extension-twist coupling. A set of composite model rotor blades was manufactured from existing blade 
molds for a low-twist helicopter rotor blade, with a view towards establishing a preliminary "proof of concept" for extension-twist-coupled rotor blades. Hover testing of the set of rotor blades was conducted with the primary goal of measuring the change in blade twist as a function of rotor speed. Data were obtained in hover for both a ballasted and unballasted blade configuration in sea level atmospheric conditions. In addition, a ballasted blade configuration was similarly tested in near-vacuum conditions, as a means of determining the effect of aerodynamic contributions to the total twist obtained. The blades were spun through the $0-800 \mathrm{rpm}$ range, with a corresponding sweep of collective pitch at $100 \mathrm{rpm}$ intervals to determine the effect on the blade elastic twist obtained. Test data were compared with results obtained from a geometrically non-linear analysis of a detailed finite-element model of the rotor blade.

\section{Apparatus and Procedures}

\section{Test Facilities}

The experimental investigation was conducted in the Helicopter Hover Facility (HHF) and the Transonic Dynamics Tunnel (TDT) located at NASA Langley. The HHF houses a hover test stand and is located adjacent to the TDT. The laboratory is located in a high bay area with intemal dimensions of $120 \mathrm{ft}$. by $75 \mathrm{ft}$. and $42 \mathrm{ft}$. high. The facility is located at an altitude of 7 feet above mean sea level. The rotor test stand is surrounded by an open-topped wire-mesh cage which is 30 feet square and $\mathbf{1 8 . 5}$ feet in height. For safety considerations, a second mesh of steel fencing and 1/4 inch steel plating is attached to the cage extending approximately 3.5 feet above and below the plane of the rotor. The center of rotation for the rotor system is located 15 feet above floor level and 24 feet from the nearest wall. Model scale rotors of up to approximately 10 feet in diameter may be tested in the HHF using the Aeroelastic Rotor Experimental System (ARES). Instrumentation in the HHF consists of ambient pressure, temperature, and relative humidity sensors. Data may be acquired and stored using the MODCOMP Data Acquisition System of the TDT.

The TDT is a continuous-flow tunnel with a slotted test section capable of operation up to a Mach number of 1.2 at stagnation pressures of $0.1 \cdot 1.0 \mathrm{atmosphere}$. The tunnel test section is 16 feet square with cropped comers and has a cross-sectional area of $248 \mathrm{ft}^{2}$. Either air or refrigerant-12 (R-12) may be used as the test medium. For this investigation, air at atmospheric pressure (with a density of $4.355 \times 10^{-5} \mathrm{lb} / \mathrm{in}^{3}$ ) was used as the test medium in a "wind-off" condition. In addition, testing was conducted in the TDT in a simulated near-vacuum condition at a static pressure of $0.403 \mathrm{lb} / \mathrm{in}^{2}$.

\section{Model Description}

A four-bladed articulated model rotor hub with coincident lead-lag and flapping hinges was used in this investigation. The hub was operated with a pitch-flap coupling ratio of 0.5 (flap up, pitch down). The attachment point of the blade pitch link was 1.40 inches aft of the blade pitch axis. One set of composite model rotor blades, manufactured from existing blade molds for a low-twist helicopter rotor blade, was tested during this investigation to establish a preliminary "proof of concept" for extension-twist-coupled rotor blades. These blades were rectangular in planform with an initial twist, $\theta_{1}$, of magnitude $-8.25^{\circ}$ (nose down) with zero twist initiating at the blade root (radial station 12.5) and decreasing linearly along the blade span. The geometric planform and cross section of the extension-twist-coupled model rotor blade are shown in figure 1 . The rotor blade measured 42.5 inches lengthwise and 4.24 inches chordwise, and incorporated a NACA 0012 airfoil design. The first 3.5 inches of the blade comprised a transition section that housed the root anchor box, which was used as a means of attaching the blade to the rotor hub fixture.

The spar, which was the primary load carrying member, was fabricated using four plies of Fiberite graphite/epoxy in a plain weave cloth "pre-preg". The $0^{\circ} / 90^{\circ}$ cloth weave was rotated off-axis to achieve a $\left(\left[+20^{\circ}\right] 4\right)$ extension-twist-coupled laminate. The trailing edge was composed of honeycomb filler wrapped by a single layer of fiberglass skin. Two hollow fiberglass weight tubes were incorporated into the blade design to provide a means of changing the non-structural mass distribution. The tubes spanned the full length of the blade and were located immediately fore and aft of the blade spar and centered about the quarter-chord. The weight tubes were closed out by stainless steel retainment tubes of smaller diameter which initiated internally two inches inboard of the blade tip (radial station 53.0).

Two different mass distributions were used in this study: a ballasted configuration in which both weight tubes were filled with 0.5 inch length rod-shaped tungsten weights, and an unballasted configuration in which both weight tubes remained empty. The two blade mass configurations were used to produce different levels of centrifugal force and thus blade twist. The core of the blade spar contained lead ballast weights, designed to balance the chordwise c.g. at the quarter-chord without any tungsten weights added. The remaining internal volume of the spar was filled with balsa, which served as a permanent mandrel in the layup process for the built-in blade twist. The aft section of the blade was filled with Nomex honeycomb to prevent crushing, as well as a small quantity of fiberglass which was used to construct the extreme trailing edge of the blade. A summary of the geometric properties of the extension-twist-coupled model rotor blades is presented in table 1 . The material and strength properties of the graphite and fiberglass materials are given in table 2, while the three-dimensional material properties of the balsa wood are defined in equation 1.

$\left(\begin{array}{c}\sigma_{x} \\ \sigma_{y} \\ \sigma_{z} \\ \tau_{x y} \\ \tau_{y z} \\ \tau_{z x}\end{array}\right)=\left[\begin{array}{rrrrrr}200,000.6146 . & 3127 . & 0 . & 0 & 0 \\ & 15012.3267 . & 0 & 0 & 0 \\ & & 4293 & 0 & 0 & 0 \\ & & & 13888 . & 0 & 0 \\ & \$ m & & & 1250 & 0 \\ & & & & & 8520 .\end{array}\right]\left(\begin{array}{c}\varepsilon_{x} \\ \varepsilon_{y} \\ \varepsilon_{z} \\ \gamma_{x y} \\ \gamma_{y z} \\ \gamma_{z x}\end{array}\right)$ 
The beam stiffnesses, weights, and inertias are presented in tables 3 and 4 for the unballasted and ballasted configurations, respectively. The properties listed in these tables are cross section properties about the principle axes. The stiffness properties are relative to the intersection of the neutral axes, which occurs at $0.30 \mathrm{c}$. Although the actual blade is twisted from $0^{\circ}$ at the blade root (station 12.5) to $-8.25^{\circ}$ at the blade tip (station 55.0 ), the properties in these tables do not reflect the blade twist.

Three of the original six blades were instrumented. Instrumentation consisted of a set of four resistance-wire Wheatstone bridge strain gauges located on the upper and lower blade surfaces at radial stations $20.75,29.45$, 41.00 , and 47.05 (figure 2). These gauges were calibrated to bending moments in the flap and chord directions, and to twist in the torsion direction. The bending moments were used to monitor blade structural integrity during the test while the twist function was used to measure the change in blade elastic twist as a function of rotor speed. The blade twist calibration was performed similar to a torsion load calibration except that the instantaneous twist rate was calculated at each bridge location during the procedure rather than the torsion load. The total elastic twist at any given location could then be obtained by integrating the twist rates from the root to the desired location. A check of the twist calibration was performed by making use of the extension-twist coupling feature of these rotor blades. An extension load was applied to the cantilevered blades and the twist rate induced by the coupling was measured at the bridge locations. The calibrated slopes of twist rate per volt were consistent with those produced by the twist calibration, indicating that the axial load had a negligible influence. The influence of blade flapwise and chordwise bending on the twist calibration was also determined to be negligible.

The set of blades was tested using the ARES model shown in figure 3. The rotor controls and drive system of the ARES are enclosed by a streamlined fuselage. The ARES is powered by a variable-frequency synchronous motor rated at 47 -hp output at $12,000 \mathrm{rpm}$. The motor is connected to the rotor shaft through a belt-driven twostage speed reduction system. The ARES rotor control system and rotor-shaft angle of attack are remotely controlled from the control room in either the TDT or the HHF. The model rotor-shaft angle of attack is varied by an electrically controlled hydraulic actuator. Blade collective pitch and lateral and longitudinal cyclic pitch are input to the rotor shaft through a swashplate, which is moved by three electrically controlled hydraulic actuators.

Instrumentation on the ARES allows continuous displays of model rotor control settings, rotor moments and forces, blade structural moments, and pitch-link loads. The ARES rotor-shaft pitch attitude is measured by a static accelerometer, and rotor control positions are measured by linear potentiometers connected to the hydraulic actuators which move the swashplate. Rotorblade flapping and lagging are measured by rotary potentiometers mounted on the rotor hub. Rotor-shaft speed is determined by a magnetic sensor. The rotatingblade data are transferred through a 30-channel slip-ring assembly. Rotor forces and moments are measured by a six-component strain gauge balance mounted below the rotor pylon and drive system. The balance is fixed with respect to the rotor shaft and pitches with the fuselage. Fuselage forces and moments are not sensed by the balance.

\section{Test Procedure}

The change in blade elastic twist as a function of rotor speed was demonstrated by spinning the rotor blades in an atmospheric hover condition for both an unballasted and ballasted blade configuration. The unballasted blade configuration, in which both weight tubes remained empty, served as a baseline with respect to blade elastic twist. The unballasted blade weighed 1.44 pounds (empty blade, no attachment hardware) and had a flapping weight of 3.16 pounds (empty blade plus attachment hardware weight). The ballasted blade configuration incorporated the full complement of 0.5 inch length tungsten weights in the fore and aft weight tubes, resulting in a 1.186 pound increase in both blade structural and flapping weights. This increase in blade weight allowed increased levels of centrifugal force to be generated along the blade span, thereby augmenting blade elastic twist.

The blades were spun through the $0-800 \mathrm{rpm}$ range at $100 \mathrm{rpm}$ intervals using a shaft angle of attack of $0^{\circ}$. At each rpm interval, a corresponding sweep of the collective pitch angle, $\theta$, was performed to determine the effect of increased lift on the blade elastic twist. The initial collective pitch angle for each rpm interval was set to the minimum necessary to obtain a zero coning condition for the blades. Data were averaged and recorded for this condition. The collective pitch was then varied in increments of $2^{\circ}$ up to a maximum pitch of $18^{\circ}$, and data were recorded at each corresponding value of collective pitch.

The blades were additionally tested in a near-vacuum condition to assess the effect of aerodynamic contributions on the total twist obtained. The blades were spun through the $0-800 \mathrm{rpm}$ range using the ballasted configuration only. Collective pitch was varied and data were recorded at pitch settings of $0^{\circ}, 7^{\circ}$, and $15^{\circ}$. Larger increments in collective pitch were used due to the negligible lift obtained in the near-vacuum condition.

All strain gauge and balance voltage readings were zeroed prior to each test run, with the non-rotating blades resting on the down stops. At each test point, tunnel parameter data were averaged and stored digitally. At the completion of each run, all strain gauge and balance voltage readings were again recorded with the non-rotating blades resting on the downstops. These final voltage readings were used to correct for any amplifier voltage drift which may have occurred during the test run.

Analysis Model

The composite rotor blade was modeled and analyzed using the MSC/NASTRAN finite-element program 
(reference 6). The finite-element model, shown in figure 4 , was composed of 381 grid points, 348 flat plate elements, and 84 solid elements. The flat plate elements were used to represent the composite spar laminate and skin, while the solid elements were used to model the balsa wood and honeycomb filler components internal to the blade structure. In addition, a total of 114 beam elements was used to represent the two internal ballast weight tubes within the blade, as well as the transition blade area (first 3.5 inches of the blade), root anchor, rotor cuff, and hub arm. The weights and densities of the structural components were calculated and verified using dissected samples taken from a sacrificed spare blade. These properties are given in table 5. Twenty-two plate elements were used circumferentially to model the airfoil cross section. The plane of grid points for each model was located at the geometric midplane of the 4-ply laminate, and was therefore coincident with the element reference plane. Structural mass was accounted for through the use of the consistent mass matrix option in the analyses. The finite-element model was analyzed for a collective pitch setting of $0^{\circ}$, measured at the blade root.

\section{Discussion of Results}

A set of extension-twist-coupled composite model rotor blades was tested as previously described. The change in blade elastic twist is plotted as a function of rotor speed for test and analysis, using both mass distributions, in figure 5 for the sea level atmospheric test condition. The data points represent twist values recorded for the lowest available collective pitch settings at each rpm interval. Test results showed maximum twists of $2.54^{\circ}$ for the unballasted blade configuration, and $5.24^{\circ}$ for the ballasted blade. These results were compared with a geometrically non-linear finite-element analysis which yielded maximum twists of $3.02^{\circ}$ for the unballasted blade configuration, and $5.61^{\circ}$ for the ballasted blade configuration.

The ballasted blades were also tested in a nearvacuum environment as a means of determining the effect of aerodynamic contributions to the total twist obtained. Figure 6 illustrates the effect of the test medium pressure on the blade elastic twist. The maximum twist obtained in the near-vacuum environment was nearly unchanged from that of the corresponding atmospheric result, differing by a maximum of $0.17^{\circ}$. These results indicate that the total twist obtained is primarily a function of the extension-twist coupling present in the rotor blade laminate and is not significantly influenced by aerodynamic loading.

The effect of blade collective pitch on the blade elastic twist is shown in figures 7 and 8 for the unballasted and ballasted blade, respectively, in the sea level atmospheric test condition. The change in blade elastic twist is plotted as a function of the thrust coefficient/solidity ratio for $200 \mathrm{rpm}$ incremental values.
Typically, an increase in blade collective pitch results in increased thrust (excluding the condition of a stalled blade). The blades will flap upward in response to this increased thrust, thereby changing the centrifugallyinduced forces acting on the blade. Because the twist is a function of centrifugal force for an extension-twistcoupled design, the magnitude of this flap angle, if significant, could diminish the effective centrifugal force acting along the blade, thereby decreasing the magnitude of twist obtained. However, no appreciable degradation in the blade elastic twist was observed for either configuration because the blade coning angle remained small throughout the sweep, reaching a maximum of only $2.5^{\circ}$ during the ballasted configuration test run.

\section{Cenclusions}

This study has demonstrated the feasibility of passive blade twist control using an extension-twist-coupled composite rotor blade design. A set of low-twist modelscale helicopter rotor blades was manufactured from existing molds, with a view towards demonstrating the passive blade twist control concept. Hover testing of the set of blades produced maximum twist changes of $5.24^{\circ}$ for the ballasted blade configuration, and $2.54^{\circ}$ for the unballasted blade configurations in the atmospheric test condition. These results compared well with those obtained from a detailed finite-element analysis model of the rotor blade, which yielded maximum twists of $3.01^{\circ}$ and $5.61^{\circ}$ for the unballasted and ballasted blade configurations, respectively. In addition, aerodynamicinduced effects on blade elastic twist were found to be minimal, with the blade twist remaining nearly unchanged between the atmospheric and near-vacuum conditions. A maximum twist difference of $0.17^{\circ}$ was observed between the two test environments. The effect of collective pitch sweep on the blade elastic twist was also demonstrated to be minimal, as no appreciable degradation in twist was observed at increased collective pitch angles.

Results from this study have contributed to the establishment of an analytical/experimental database for elastically-coupled structures. Future studies of increased scope and complexity, including the investigation of the effect of large values of pre-twist in elastically-coupled rotor blades, will further contribute to this database and provide a basis for the development of practical composite tiltrotor blade designs incorporating extensiontwist coupling. 


\section{References}

1. Kosmatka, J. B.: Extension-Bend-Twist Coupling Behavior of Nonhomogeneous Anisotropic Beams with Initial Twist, Proceedings of the AIAA 32nd Structures, Structural Dynamics and Materials Conference, Baltimore, MD, April 8-10, 1991.

2. Nixon, M. W. : Analytical and Experimental Investigations of Extension-Twist-Coupled Structures, Master's thesis submitted to the School of Engineering and Applied Science, George Washington University, May 1989.

3. Minguet, P., and Dugundji, J.: Experiments and Analysis for Composite Blades Under Large
Deflections, Part 1 - Static Behavior, AIAA Journal, Vol. 28, No. 9, September, 1990, pp. 1573-1579.

4. Nixon, M. W. : Extension-Twist Coupling of Composite Circular Tubes with Application to Tilt Rotor Blade Design, Proceedings of the AIAA 28th Structures, Structural Dynamics and Materials Conference, Monterey, CA, April 6-8, 1987, pp. 295-303.

5. Nixon, M. W. : Improvements to Tilt Rotor Performance Through Passive Blade Twist Control, NASA TM-100583, AVSCOM TM-88-B-010, April 1988.

6. MSC/NASTRAN User's Manual-Version 64, MSR39, MacNeal-Schwendler Corporation, July 1984.
Rotor Type

Number of Blades, $b$

Rotor Diameter

Blade Chord, $c$

Solidity, $\sigma$

Airfoil Section

Theoretical Blade Twist about $c / 4$

Hinge Offset, Flap and Lead-Lag Coincident

Root Cutout

Blade Elastic Axis

Blade Pitch Axis

Center of Gravity

Blade Weight (without root)

Blade Flapping Weight (with root and cuff)

Blade First Mass Moment (with root and cuff) about flap hinge

Blade Second Mass Moment (with root and cuff) about flap hinge
Fully Articulated

4

110 in

4.24 in

0.0982

NACA 0012

$-8.25^{\circ}$ linear from $r=12.5$ to $r=55.0$ in

$r=3.0$ in

$r=12.5$ in

$25 \%$ Chord nominally

25\% Chord

24\% Chord, no ballast

$23 \%$ Chord, full ballast

$1.44 \mathrm{lb}$, no ballast

$2.60 \mathrm{lb}$, full ballast

$3.16 \mathrm{lb}$, no ballast

$4.32 \mathrm{lb}$, full ballast

0.1365 slug-ft, no ballast

0.2263 slug- $\mathrm{ft}$, full ballast

0.3319 slug- $\mathrm{ft}^{2}$, no ballast

0.5856 slug- $\mathrm{ft}^{2}$, full ballast

Table 1 - Properties of the extension-twist-coupled research model rotor system.

\begin{tabular}{|c|c|c|c|c|c|c|c|}
\hline Material & $\begin{array}{c}E_{11} \\
\left(l b / i^{2}\right)\end{array}$ & $\begin{array}{c}E_{22} \\
\left(l b / i^{2}\right)\end{array}$ & $v_{12}$ & $\begin{array}{c}G_{12} \\
\left(l b / i^{2}\right)\end{array}$ & $\begin{array}{l}X_{T}, X_{C} \\
\left(l b / i^{2}\right)\end{array}$ & $\begin{array}{l}Y_{T}, Y_{C} \\
\left(l b / i^{2}\right) \\
\end{array}$ & $\begin{array}{c}S \\
\left(l b / i^{2}\right)\end{array}$ \\
\hline $\begin{array}{l}\text { Fiberite (fabric) } \\
\text { Glass (fabric) } \\
\text { Graphite (uni-tape) }\end{array}$ & $\begin{array}{r}10.50 \mathrm{E} 6 \\
3.50 \mathrm{E} 6 \\
15.00 \mathrm{E} 6 \\
\end{array}$ & $\begin{array}{r}10.50 \mathrm{E} 6 \\
3.50 \mathrm{E} 6 \\
0.45 \mathrm{E} 6\end{array}$ & $\begin{array}{l}0.077 \\
0.099 \\
0.280\end{array}$ & $\begin{array}{l}0.95 \mathrm{E} 6 \\
0.40 \mathrm{E} 6 \\
0.90 \mathrm{E} 6 \\
\end{array}$ & $\begin{array}{r}12.54 \mathrm{E} 4 \\
9.00 \mathrm{E} 4 \\
21.10 \mathrm{E} 4 \\
\end{array}$ & $\begin{array}{r}12.54 \mathrm{E} 4 \\
9.00 \mathrm{E} 4 \\
6.10 \mathrm{E} 3 \\
\end{array}$ & $\begin{array}{r}15.88 \mathrm{E} 3 \\
8.54 \mathrm{E3} \\
13.80 \mathrm{E} 3 \\
\end{array}$ \\
\hline
\end{tabular}

Table 2 - Material stiffness and strength properties. 


\begin{tabular}{|r|cccccccccc|}
\hline $\begin{array}{c}\text { Section, } r \\
\text { (in })\end{array}$ & $\begin{array}{c}C_{11} \\
(l b)\end{array}$ & $\begin{array}{c}C_{22} \\
(l b)\end{array}$ & $\begin{array}{c}C_{33} \\
(l b)\end{array}$ & $\begin{array}{c}C_{44} \\
\left(l b-i n^{2}\right)\end{array}$ & $\begin{array}{c}C_{55} \\
\left(l b-i n^{2}\right)\end{array}$ & $\begin{array}{c}C_{66} \\
\left(l b-i n^{2}\right)\end{array}$ & $\begin{array}{c}C_{14} \\
(l b-i n)\end{array}$ & $\begin{array}{c}C_{25} \\
(l b-i n)\end{array}$ & $\begin{array}{c}C_{36} \\
(l b-i n)\end{array}$ & $\begin{array}{c}I_{\theta} \\
\left(l b-s^{2}\right)\end{array}$ \\
\hline $6.9-12.5$ & $10.50 \mathrm{E} 6$ & $3.89 \mathrm{E} 6$ & $3.89 \mathrm{E} 6$ & $1.46 \mathrm{E} 6$ & $2.77 \mathrm{E} 5$ & $3.67 \mathrm{E} 6$ & 0. & 0. & 0. & $1.00 \mathrm{E}-4$ \\
$12.5-14.0$ & $3.64 \mathrm{E} 6$ & $1.35 \mathrm{E} 6$ & $1.35 \mathrm{E} 6$ & $7.05 \mathrm{E} 6$ & $3.75 \mathrm{E} 4$ & $3.38 \mathrm{E} 5$ & 0. & 0. & 0. & $3.58 \mathrm{E}-4$ \\
$14.0-16.0$ & $6.33 \mathrm{E} 6$ & $2.12 \mathrm{E} 6$ & $2.12 \mathrm{E} 6$ & $1.25 \mathrm{E} 5$ & $9.75 \mathrm{E} 4$ & $2.09 \mathrm{E} 7$ & $-8.80 \mathrm{E} 4$ & $-2.08 \mathrm{E} 5$ & $6.97 \mathrm{E} 4$ & $3.58 \mathrm{E}-4$ \\
$16.0-20.0$ & $3.00 \mathrm{E} 6$ & $9.90 \mathrm{E} 5$ & $1.40 \mathrm{E} 5$ & $8.50 \mathrm{E} 4$ & $9.47 \mathrm{E} 4$ & $1.38 \mathrm{E} 6$ & $-8.80 \mathrm{E} 4$ & $-2.08 \mathrm{E} 5$ & $6.97 \mathrm{E} 4$ & $5.88 \mathrm{E}-5$ \\
$20.0-53.0$ & $2.62 \mathrm{E} 6$ & $9.71 \mathrm{E} 5$ & $1.03 \mathrm{E} 5$ & $3.25 \mathrm{E} 4$ & $7.44 \mathrm{E} 4$ & $1.21 \mathrm{E} 6$ & $-8.80 \mathrm{E} 4$ & $-2.08 \mathrm{E} 5$ & $6.97 \mathrm{E} 4$ & $5.05 \mathrm{E}-5$ \\
$53.0-55.0$ & $3.57 \mathrm{E} 6$ & $1.33 \mathrm{E} 6$ & $4.67 \mathrm{E} 5$ & $3.41 \mathrm{E} 4$ & $7.64 \mathrm{E} 4$ & $2.08 \mathrm{E} 6$ & $-8.80 \mathrm{E} 4$ & $-2.08 \mathrm{E} 5$ & $6.97 \mathrm{E} 4$ & $5.36 \mathrm{E}-5$ \\
\hline
\end{tabular}

Table 3 - Beam stiffnesses, weights, and inertias of unballasted rotor blade.

\begin{tabular}{|r|cccccccccc|}
\hline $\begin{array}{c}\text { Section, } r \\
(i n)\end{array}$ & $\begin{array}{c}C_{1 l} \\
(l b)\end{array}$ & $\begin{array}{c}C_{22} \\
(l b)\end{array}$ & $\begin{array}{c}C_{33} \\
(l b)\end{array}$ & $\begin{array}{c}C_{44} \\
\left(l b-i n^{2}\right)\end{array}$ & $\begin{array}{c}C_{55} \\
\left(l b-i n^{2}\right)\end{array}$ & $\begin{array}{c}C_{66} \\
\left(l b-i n^{2}\right)\end{array}$ & $\begin{array}{c}C_{14} \\
(l b-i n)\end{array}$ & $\begin{array}{c}C_{25} \\
(l b-i n)\end{array}$ & $\begin{array}{c}C_{36} \\
(l b-i n)\end{array}$ & $\begin{array}{c}I_{\theta} \\
\left(l b-s^{2}\right)\end{array}$ \\
\hline $6.9-12.5$ & $10.50 \mathrm{E} 6$ & $3.89 \mathrm{E} 6$ & $3.89 \mathrm{E} 6$ & $1.46 \mathrm{E} 6$ & $2.77 \mathrm{E} 5$ & $3.67 \mathrm{E} 6$ & 0. & 0. & 0. & $1.00 \mathrm{E}-4$ \\
$12.5-14.0$ & $3.64 \mathrm{E} 6$ & $1.35 \mathrm{E} 6$ & $1.35 \mathrm{E} 6$ & $7.05 \mathrm{E} 6$ & $3.75 \mathrm{E} 4$ & $3.38 \mathrm{E} 5$ & 0. & 0. & 0. & $4.22 \mathrm{E}-4$ \\
$14.0-16.0$ & $6.33 \mathrm{E} 6$ & $2.12 \mathrm{E} 6$ & $2.12 \mathrm{E} 6$ & $1.25 \mathrm{E} 5$ & $9.75 \mathrm{E} 4$ & $2.09 \mathrm{E} 7$ & $-8.80 \mathrm{E} 4$ & $-2.08 \mathrm{E} 5$ & $6.97 \mathrm{E} 4$ & $4.22 \mathrm{E}-4$ \\
$16.0-20.0$ & $3.00 \mathrm{E} 6$ & $9.90 \mathrm{E} 5$ & $1.40 \mathrm{E} 5$ & $8.50 \mathrm{E} 4$ & $9.47 \mathrm{E} 4$ & $1.38 \mathrm{E} 6$ & $-8.80 \mathrm{E} 4$ & $-2.08 \mathrm{E} 5$ & $6.97 \mathrm{E} 4$ & $1.23 \mathrm{E}-4$ \\
$20.0-53.0$ & $2.62 \mathrm{E} 6$ & $9.71 \mathrm{E} 5$ & $1.03 \mathrm{E} 5$ & $3.25 \mathrm{E} 4$ & $7.44 \mathrm{E} 4$ & $1.21 \mathrm{E} 6$ & $-8.80 \mathrm{E} 4$ & $-2.08 \mathrm{E} 5$ & $6.97 \mathrm{E} 4$ & $1.15 \mathrm{E}-4$ \\
$53.0-55.0$ & $3.57 \mathrm{E} 6$ & $1.33 \mathrm{E} 6$ & $4.67 \mathrm{E} 5$ & $3.41 \mathrm{E} 4$ & $7.64 \mathrm{E} 4$ & $2.08 \mathrm{E} 6$ & $-8.80 \mathrm{E} 4$ & $-2.08 \mathrm{E} 5$ & $6.97 \mathrm{E} 4$ & $5.36 \mathrm{E}-5$ \\
\hline
\end{tabular}

Table 4 - Beam stiffnesses, weights, and inertias of ballasted rotor blade.

\begin{tabular}{|l|ccccc|}
\hline \hline \multicolumn{1}{|c|}{ Component } & $\begin{array}{c}\text { Weight } \\
(\text { lb })\end{array}$ & $\begin{array}{c}\text { Length } \\
(\text { in })\end{array}$ & $\begin{array}{c}\text { Area } \\
\left(\text { in }^{2}\right)\end{array}$ & $\begin{array}{c}\rho \\
\left(l b / i^{3}\right)\end{array}$ & $\begin{array}{c}\text { Weight/hength } \\
(l b / i n)\end{array}$ \\
\hline Root section (12.5-16.0) & 0.2198 & 3.50 & - & - & 0.0628 \\
Cross section $(16.0-18.0)$ & 0.0705 & 2.00 & - & - & 0.0352 \\
Cross section (18.0-53.0) & 1.0804 & 35.0 & - & - & 0.0309 \\
Cross section (53.0-55.0) & 0.0738 & 2.0 & - & - & 0.0369 \\
Trailing edge filler & 0.0013 & 2.0 & 0.0035 & 0.1886 & 0.0007 \\
Honeycomb & 0.0027 & 2.0 & 0.5431 & 0.0024 & 0.0013 \\
Aft weight tube & 0.0024 & 2.0 & - & - & 0.0012 \\
Balsa core & 0.0043 & 2.0 & 0.6461 & 0.0034 & 0.0022 \\
Fiberglass skin & - & - & 0.0415 & 0.0740 & 0.0031 \\
\hline
\end{tabular}

Table 5 - Weights and densities calculated from blade samples. 

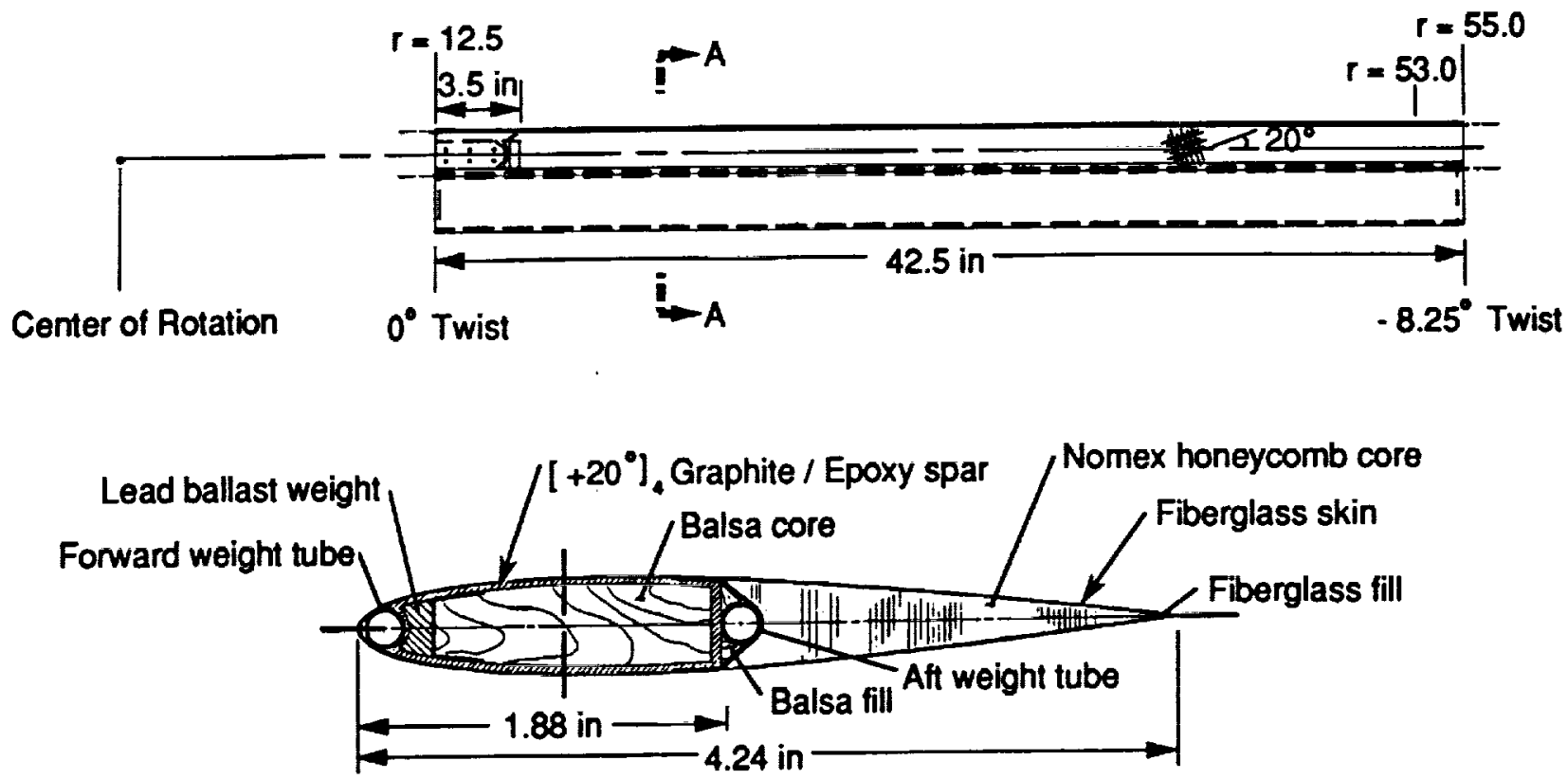

Section A-A

Figure 1 - Extension-twist-coupled model rotor blade geometry.

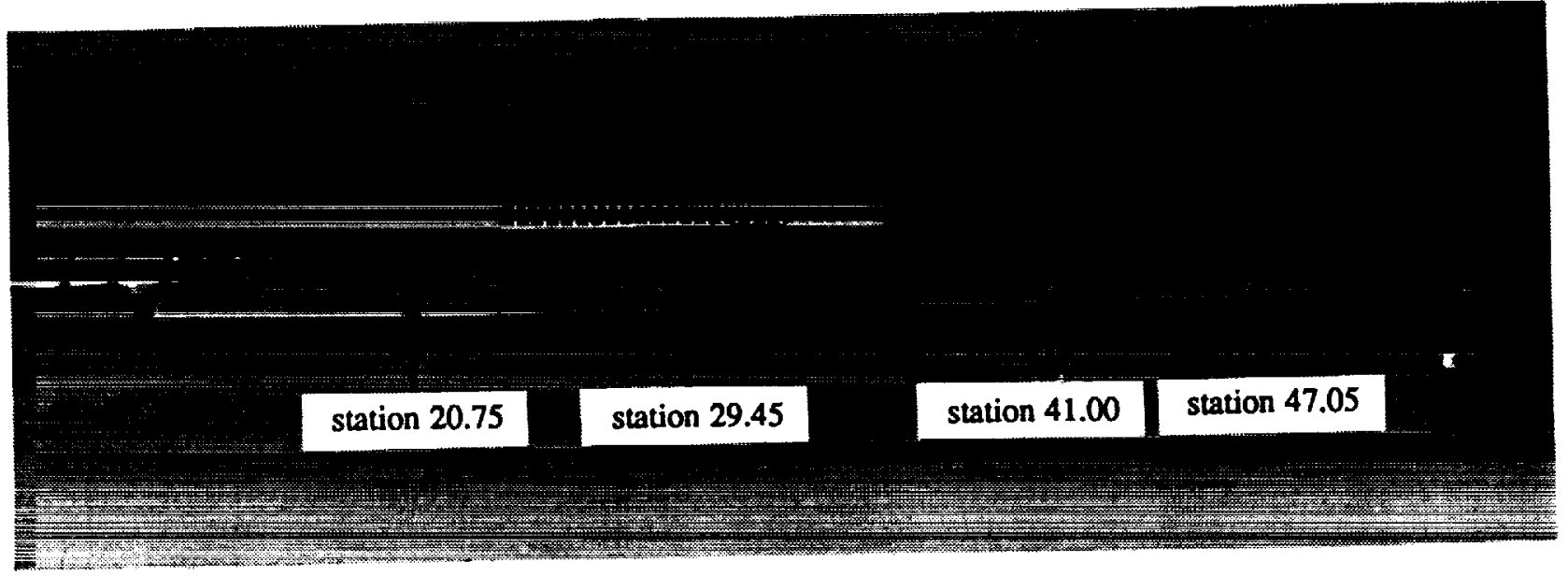

Figure 2 - Layout of twist measuring instrumentation. 


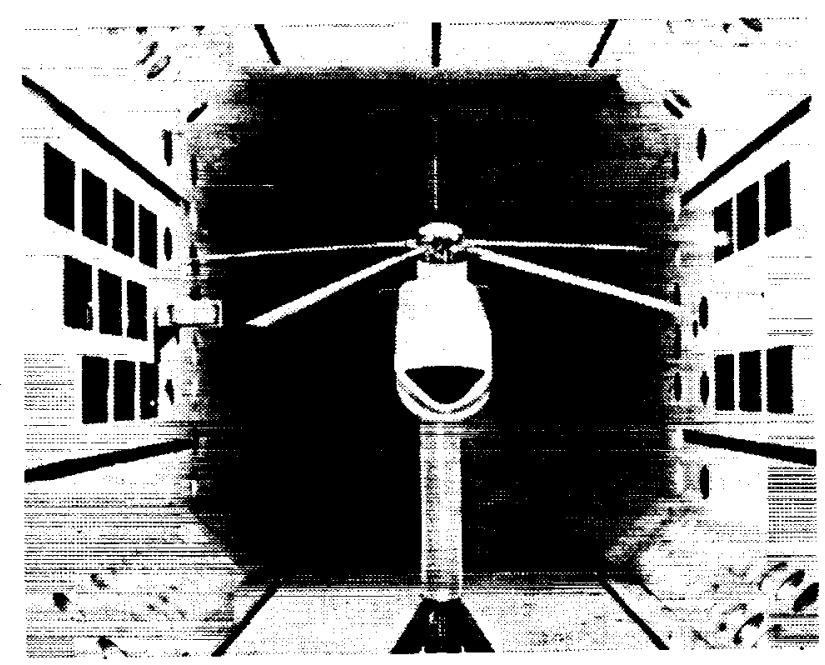

Figure 3 - Aeroelastic Rotor Experimental System (ARES) model in the Transonic Dynamics Tunnel.

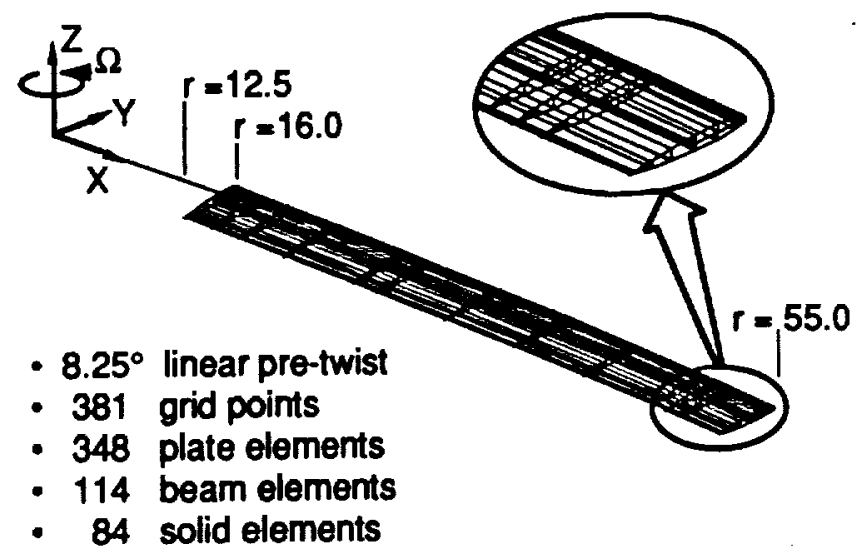

Figure 4 - Finite-element model of extension-twist-coupled model rotor blade.

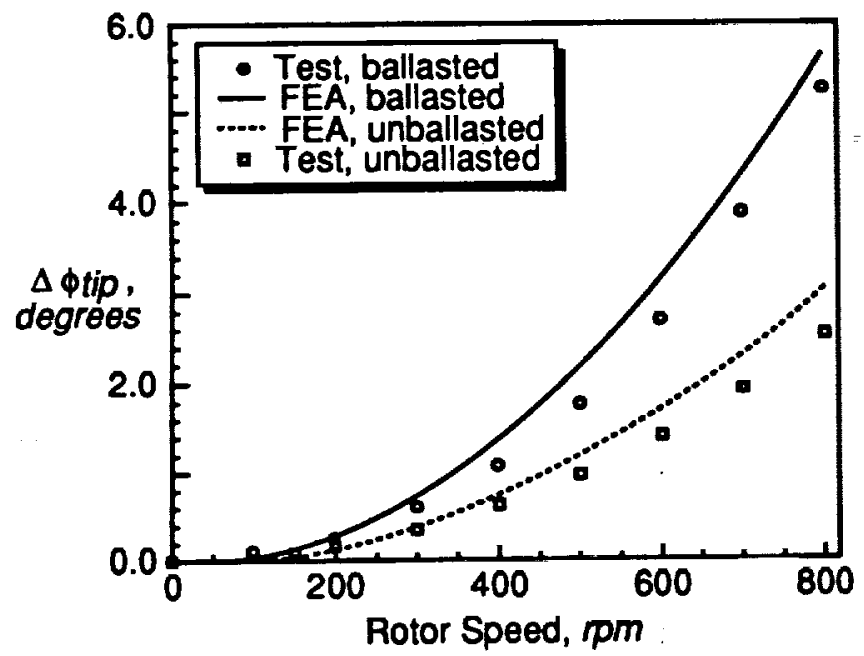

Figure 5 - Change in blade elastic twist at tip as a function of rotor speed.

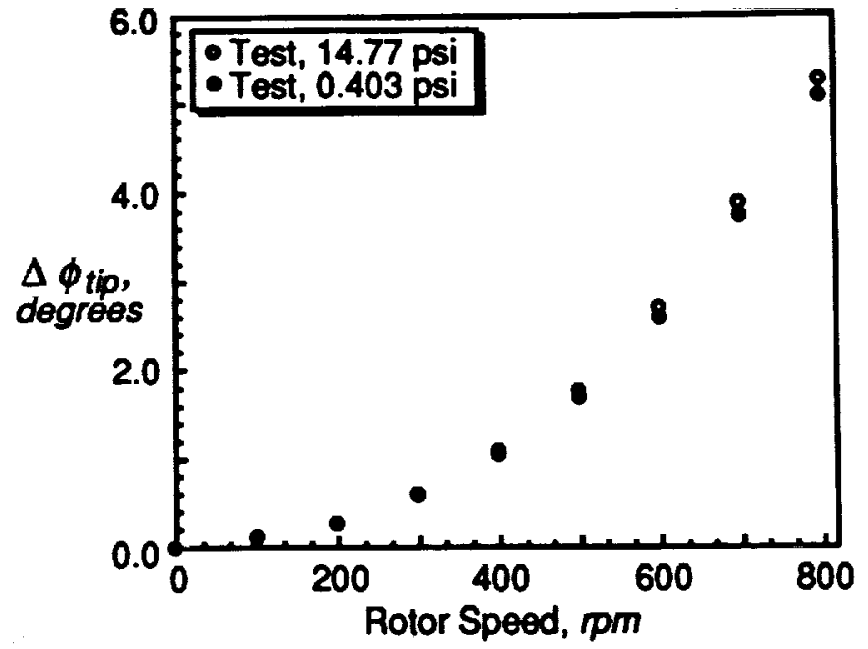

Figure 6 - Effect of test medium pressure on ballasted blade elastic twist at tip.

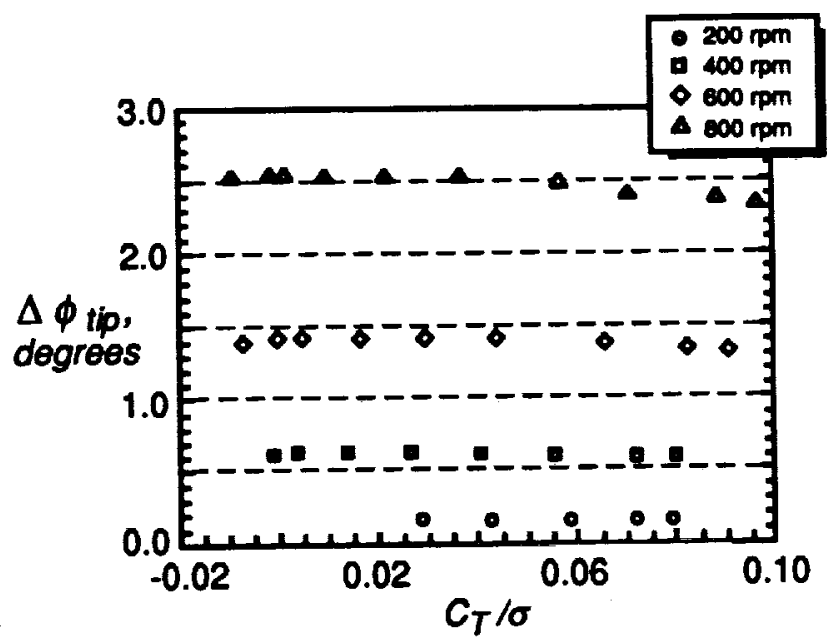

Figure 7 - Blade elastic twist as a function of thrust coefficient /solidity ratio for unballasted blade.

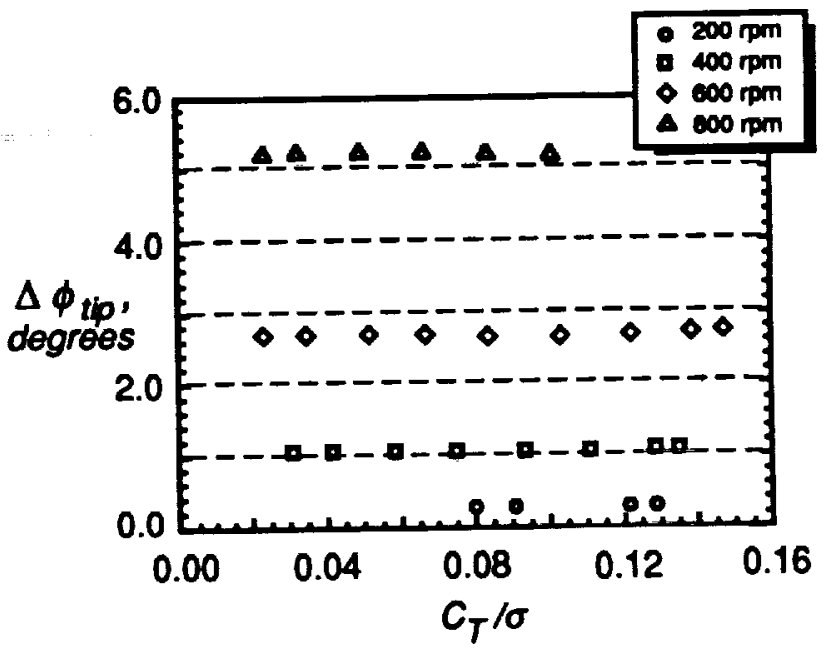

Figure 8 - Blade elastic twist as a function of thrust coefficient /solidity ratio for ballasted blade. 


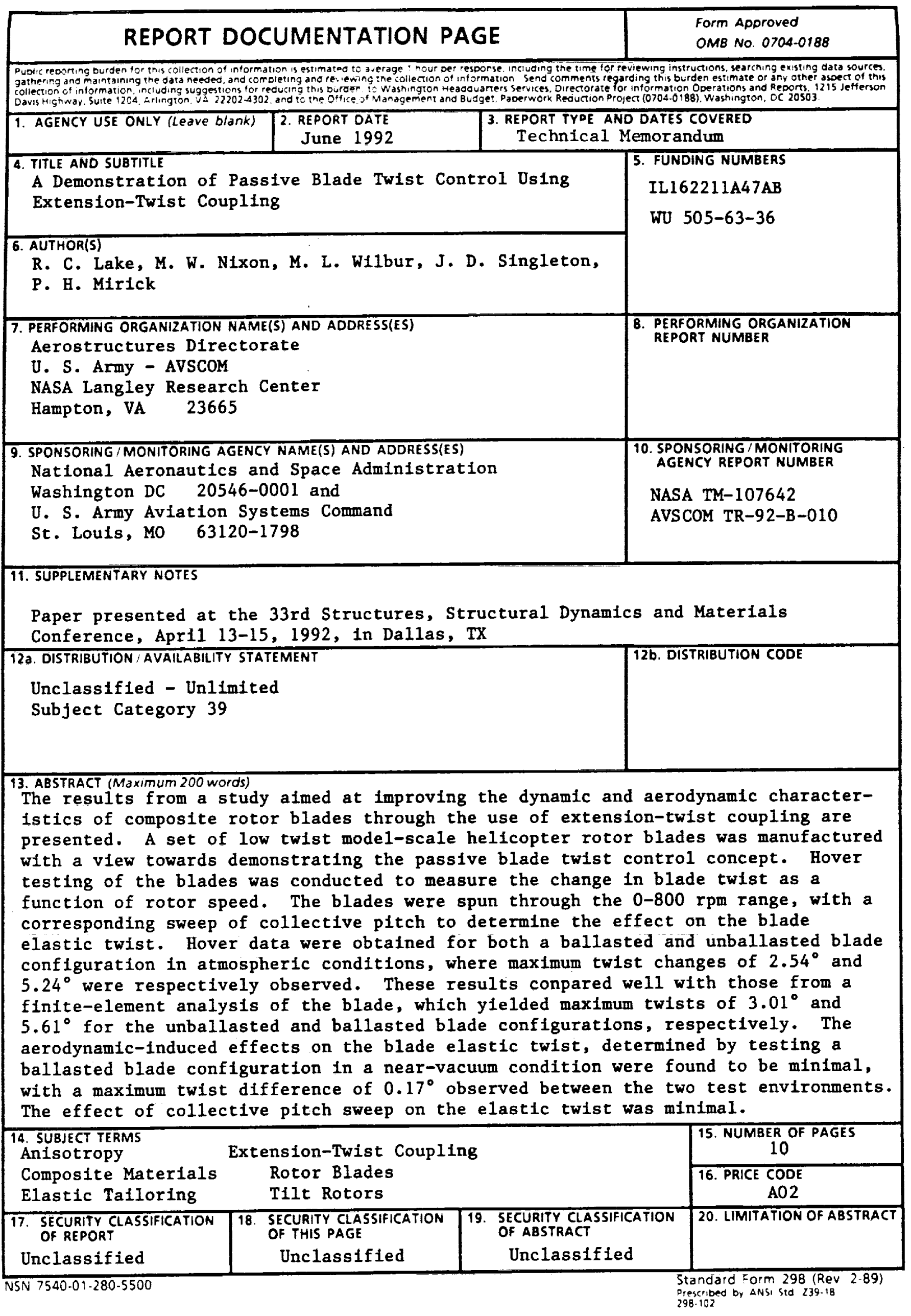

